\title{
A NOTE ON SOME DIOPHANTINE EQUATIONS
}

\author{
REFIK KESKINN AND MERVE GÜNEY DUMAN
}

Received 05 November, 2015

\begin{abstract}
Let $k \geq 3$ be an odd integer. In this paper we investigate all positive integer solutions of the equations $x^{4}-k x^{2} y+y^{2}=\mp A, x^{4}-k x^{2} y+y^{2}=\mp A\left(k^{2}-4\right), x^{4}-\left(k^{2}-4\right) y^{2}=\mp 4 A$, and $x^{2}-\left(k^{2}-4\right) y^{4}=\mp 4 A$ with $A=\mp(k \mp 2)$. We show that if $k \equiv 1(\bmod 8)$ and $k^{2}-4$ be a square-free integer, then the equation $x^{4}-k x^{2} y+y^{2}=(k-2)\left(k^{2}-4\right)$ has no positive integer solutions. Moreover, if $k^{2}-4$ be a square-free integer, then the equation $x^{4}-k x^{2} y+y^{2}=$ $-(k+2)\left(k^{2}-4\right)$ has no positive integer solutions.
\end{abstract}

2010 Mathematics Subject Classification: 11B37; 11B39

Keywords: generalized Fibonacci numbers, generalized Lucas numbers, Diophantine equations

\section{INTRODUCTION}

Let $k$ and $s$ be two nonzero integers with $k^{2}+4 s>0$. The generalized Fibonacci sequence is defined by $U_{0}(k, s)=0, U_{1}(k, s)=1$, and $U_{n+1}(k, s)=k U_{n}(k, s)+$ $s U_{n-1}(k, s)$ for $n \geqslant 1$ and generalized Lucas sequence is defined by $V_{0}(k, s)=2$, $V_{1}(k, s)=k$, and $V_{n+1}(k, s)=k V_{n}(k, s)+s V_{n-1}(k, s)$ for $n \geqslant 1$, respectively. Moreover, generalized Fibonacci and Lucas numbers for negative subscripts are defined as $U_{-n}=-(-s)^{-n} U_{n}$ and $V_{-n}=(-s)^{-n} V_{n}$ for $n \in \mathbb{N}$. Especially $U_{-n}(k,-1)=-U_{n}(k,-1)$ and $V_{-n}(k,-1)=V_{n}(k,-1)$ for every natural number $n$.

In the literature, there are many papers dealing with the number of the solutions of the equation $a x^{4}+b x^{2} y+c y^{2}-d=0$. In [7], the author investigated all nonnegative integer solutions of the equations $x^{4}-k x^{2} y+y^{2}=1,4$, under the assumption that $k$ is even. If $k \neq 318$ and $k$ is not a perfect square, then all nonnegative integer solutions of the equation $x^{2}-k x y^{2}+y^{4}=1$ are $(x, y)=(k, 1)$, $(1,0),(0,1)$. If $k=338$, then all positive integer solutions of this equation are $(x, y)=(13051348805,6214),(114243,6214)$. If $k$ is a perfect square, then all positive integer solutions of this equation are $(x, y)=(1, \sqrt{k}),\left(k^{2}-1, \sqrt{k}\right)$. Moreover, the author showed that if $k=2 v^{2}$ for some integer $v$, then all positive integer solutions of the equation $x^{2}-k x y^{2}+y^{4}=4$ are $(x, y)=(2, \sqrt{2 k}),\left(2 k^{2}-2, \sqrt{2 k}\right)$.

The second author was supported in part by the TÜBİTAK. 
Otherwise, the only nonnegative integer solution of this equation is $(x, y)=(2,0)$. In [2], wherein the author investigated all positive integer solutions of the equation $x^{2}-k x y^{2}+y^{4}=c$ for $c \in\{\mp 1, \mp 2, \mp 4\}$, with the assumption that $k$ is odd for the particular cases $c=1$ or 4 . He showed that the equation $x^{2}-k x y^{2}+y^{4}=4$ has no positive integer solutions. Moreover, he showed that if $y=k^{2}$ for some odd integer $k$, then the only integer solution of the equation $x^{2}-k x y^{2}+y^{4}=1$ is $y=1$. Otherwise, all positive integer solutions of this equation are $y=1$ or $k$. Moreover, he showed that the equation

$$
x^{2}-k x y^{2}+y^{4}=-1
$$

has no integer solutions when $k=3$. Otherwise, the only integer solution of the equation (1.1) is $y=1$. In addition, he showed that the equation

$$
x^{2}-k x y^{2}+y^{4}=-4
$$

has a solution only when $k=3$ or 6 . If $k=3$, then all positive integer solutions of equation (1.2) are given by $(x, y)=(2,2)$ or $(10,2)$. If $k=6$, then all positive integer solutions of equation $(1.2)$ are given by $(x, y)=(1,1),(5,1),(29,13)$ or $(985,13)$.

Let $k$ be an odd integer. In this paper, firstly, we solve the equations $x^{2}=$ $V_{n}(k,-1) \mp V_{n-1}(k,-1)$ and $x^{2}=U_{n}(k,-1) \mp U_{n-1}(k,-1)$, respectively. We show that if $k \equiv 3(\bmod 8)$, then the equation $x^{2}=V_{n}(k,-1)+V_{n-1}(k,-1)$ has no solutions and if $k \equiv 1(\bmod 8)$, then the equation $x^{2}=V_{n}(k,-1)-V_{n-1}(k,-1)$ has no solutions. Lastly, we find all positive integer solutions of the following equations

$$
\begin{aligned}
& x^{4}-\left(k^{2}-4\right) y^{2}=4(k+2), \\
& x^{4}-\left(k^{2}-4\right) y^{2}=-4(k-2), \\
& x^{2}-\left(k^{2}-4\right) y^{4}=4(k+2), \\
& x^{2}-\left(k^{2}-4\right) y^{4}=-4(k-2), \\
& x^{4}-k x^{2} y+y^{2}=-(k-2),
\end{aligned}
$$

and

$$
x^{4}-k x^{2} y+y^{2}=k+2 .
$$

We show that if $k \equiv 1(\bmod 8)$, then the equation $x^{4}-\left(k^{2}-4\right) y^{2}=-4(k-2)$ has no integer solutions. Moreover, if $k^{2}-4$ is square-free, then we investigate all positive integer solutions of the equations

$$
x^{4}-k x^{2} y+y^{2}=\left(k^{2}-4\right)(k-2)
$$

and

$$
x^{4}-k x^{2} y+y^{2}=-\left(k^{2}-4\right)(k+2) .
$$

We show that if $k \equiv 1(\bmod 8)$ and $k^{2}-4$ is square-free, then the equation $x^{4}-$ $k x^{2} y+y^{2}=(k-2)\left(k^{2}-4\right)$ has no positive integer solutions. Moreover, if $k^{2}-4$ 
is square-free, then we show that the equation $x^{4}-k x^{2} y+y^{2}=-(k+2)\left(k^{2}-4\right)$ has no positive integer solutions. Let $\left(\frac{a}{b}\right)$ represent Jacobi symbol. Then

$$
\left(\frac{a^{2}}{k}\right)=1
$$

for every $a \in \mathbb{Z}$. Moreover, we have

$$
\begin{aligned}
& \left(\frac{-1}{k}\right)=1 \text { if and only if } k \equiv 1,5(\bmod 8), \\
& \left(\frac{2}{k}\right)=1 \text { if and only if } k \equiv 1,7(\bmod 8)
\end{aligned}
$$

and

$$
\left(\frac{-2}{k}\right)=1 \text { if and only if } k \equiv 1,3(\bmod 8)
$$

\section{Preliminary Results}

From now on, $\square$ represents a perfect square. Now we give the following lemma from [5].

Lemma 1. Let $k$ be odd. If $V_{n}(k,-1)=\square$ for some natural number $n$, then $n=1$ and $k$ is a perfect square.

The following two theorems are given in [3].

Theorem 1. Let $k \geq 3$ be an odd integer. If $V_{n}(k,-1)=k \square$ for some natural number $n$, then $n=1$.

Theorem 2. Let $k \geq 3$ be an odd integer. If $V_{n}(k,-1)=q \square$ for some $q \mid k$ with $q>1$ and $n \geq 1$, then $n=1$.

The following theorem is given in [6].

Theorem 3. Let $n \in \mathbb{N} \cup\{0\}, m, r \in \mathbb{Z}$, and $m$ be a nonzero integer. Then

$$
\begin{gathered}
U_{2 m n+r} \equiv U_{r}\left(\bmod U_{m}\right), \\
U_{2 m n+r} \equiv(-1)^{n} U_{r}\left(\bmod V_{m}\right), \\
V_{2 m n+r} \equiv V_{r}\left(\bmod U_{m}\right),
\end{gathered}
$$

and

$$
V_{2 m n+r} \equiv(-1)^{n} V_{r}\left(\bmod V_{m}\right),
$$

where $U_{n}=U_{n}(k,-1)$ and $V_{n}=V_{n}(k,-1)$. 
When $k$ is odd, it is seen that $V_{2^{r}} \equiv 7(\bmod 8)$ and thus

$$
\left(\frac{-1}{V_{2^{r}}}\right)=-1
$$

for $r \geq 1$. It can be easily seen that

$$
\left(\frac{k+1}{V_{2^{r}}}\right)=\left(\frac{k-1}{V_{2^{r}}}\right)=1
$$

for $r \geq 1$. Moreover, it can be shown that if $k \equiv 1,7(\bmod 8)$, then

$$
\left(\frac{k+2}{V_{2} r}\right)=1
$$

and

$$
\left(\frac{k-2}{V_{2^{r}}}\right)=\left\{\begin{array}{cc}
-1 & \text { if } k \equiv 1,7(\bmod 8) \\
1 & \text { if } k \equiv 3(\bmod 8)
\end{array}\right.
$$

From now on, instead of $U_{n}(k,-1)$ and $V_{n}(k,-1)$, we will write $U_{n}$ and $V_{n}$, respectively. The following seven theorems are given in [4].

Theorem 4. Let $k \geq 3$ be an integer and $k+2$ be not a perfect square. Then all positive integer solutions of the equation $x^{2}-k x y+y^{2}=k+2$ are given by $(x, y)=\left(U_{n+1}+U_{n}, U_{n}+U_{n-1}\right)$ with $n \geq 1$.

Theorem 5. Let $k \geq 3$. Then all positive integer solutions of the equation $x^{2}-$ $k x y+y^{2}=-(k-2)$ are given by $(x, y)=\left(U_{n+1}-U_{n}, U_{n}-U_{n-1}\right)$ with $n \geq 0$.

Theorem 6. Let $k \geq 3$ an integer and $k+2$ be not a perfect square. Then all positive integer solutions of the equation $x^{2}-\left(k^{2}-4\right) y^{2}=4(k+2)$ are given by $(x, y)=\left(V_{n}+V_{n-1}, U_{n}+U_{n-1}\right)$ with $n \geq 1$.

Theorem 7. Let $k \geq 3$ an integer and $k+2$ be a perfect square. Then all positive integer solutions of the equation $x^{2}-\left(k^{2}-4\right) y^{2}=4(k+2)$ are given by $(x, y)=$ $\left(\sqrt{k+2} V_{n-2}, U_{n-2}\right)$ with $n \geq 1$.

Theorem 8. Let $k \geq 3$. Then all positive integer solutions of the equation $x^{2}-$ $\left(k^{2}-4\right) y^{2}=-4(k-2)$ are given by $(x, y)=\left(V_{n}-V_{n-1}, U_{n}-U_{n-1}\right)$ with $n \geq 1$.

Theorem 9. Let $k \geq 3$ an integer and $k^{2}-4$ be square-free. Then all positive integer solutions of the equation $x^{2}-k x y+y^{2}=-(k+2)\left(k^{2}-4\right)$ are given by $(x, y)=\left(V_{n+1}+V_{n}, V_{n}+V_{n-1}\right)$ with $n \geq 0$.

Theorem 10. Let $k \geq 3$ an integer and $k^{2}-4$ be square-free. Then all positive integer solutions of the equation $x^{2}-k x y+y^{2}=(k-2)\left(k^{2}-4\right)$ are given by $(x, y)=\left(V_{n+1}-V_{n}, V_{n}-V_{n-1}\right)$ with $n \geq 1$. 


\section{SOME THEOREMS AND LEMMAS}

From now on, we will assume that $n \geq 0$ and $k \geq 3$ is odd.

Lemma 2. If $k \equiv 3(\bmod 8)$, then $\left(\frac{k^{2}-k-1}{V_{2} r}\right)=-1$.

Proof. An induction method shows that

$$
V_{2^{r}} \equiv\left\{\begin{array}{cc}
k-1\left(\bmod k^{2}-k-1\right) & \text { if } r \text { is even, } \\
-k\left(\bmod k^{2}-k-1\right) & \text { if } r \text { is odd }
\end{array}\right.
$$

Let $r$ be even. Then we obtain

$$
\begin{aligned}
\left(\frac{k^{2}-k-1}{V_{2} r}\right) & =(-1)^{\left(\frac{k^{2}-k-2}{2}\right)\left(\frac{V_{2} r-1}{2}\right)}\left(\frac{V_{2^{r}}}{k^{2}-k-1}\right) \\
& =\left(\frac{k-1}{k^{2}-k-1}\right)=\left(\frac{2}{k^{2}-k-1}\right)\left(\frac{(k-1) / 2}{k^{2}-k-1}\right) \\
& =(-1)^{\frac{\left(k^{2}-k-1\right)^{2}-1}{8}}(-1)^{\left(\frac{k^{2}-k-2}{2}\right)\left(\frac{k-3}{4}\right)}\left(\frac{k^{2}-k-1}{(k-1) / 2}\right) \\
& =(-1)\left(\frac{-1}{(k-1) / 2}\right)=(-1)(-1)^{\frac{k-3}{4}}=-1 .
\end{aligned}
$$

Let $r$ be odd. Then we have

$$
\begin{aligned}
& \left(\frac{k^{2}-k-1}{V_{2^{r}}}\right)=(-1)^{\left(\frac{k^{2}-k-2}{2}\right)\left(\frac{V_{2^{r}-1}}{2}\right)}\left(\frac{V_{2^{r}}}{k^{2}-k-1}\right) \\
& =\left(\frac{-k}{k^{2}-k-1}\right)=\left(\frac{-1}{k^{2}-k-1}\right)\left(\frac{k}{k^{2}-k-1}\right) \\
& =(-1)^{\frac{k^{2}-k-2}{2}}(-1)^{\left(\frac{k^{2}-k-2}{2}\right)\left(\frac{k-1}{2}\right)}\left(\frac{k^{2}-k-1}{k}\right) \\
& =\left(\frac{-1}{k}\right)=(-1)^{\left(\frac{k-1}{2}\right)}=-1 \text {. }
\end{aligned}
$$

Since the proof of the following lemma is easy, we omit it.

Lemma 3. $V_{2^{r}} \equiv\left\{\begin{array}{cl}k\left(\bmod k^{2}+k-1\right) & \text { if } r \text { is even, } \\ -k-1\left(\bmod k^{2}+k-1\right) & \text { if } r \text { is odd. }\end{array}\right.$

By using the above lemma, we can give the following lemma.

Lemma 4. If $k \equiv 1,5(\bmod 8)$, then $\left(\frac{k^{2}+k-1}{V_{2} r}\right)=1$.

Theorem 11. Let $k \equiv 1,5,7(\bmod 8)$. If the equation $x^{2}=V_{n}+V_{n-1}$ has a solution, then $n=0$ or 1 . If $k \equiv 3(\bmod 8)$, then we have two solutions $(n, k)=(3,3)$, $(3,43)$. 
Proof. Assume that $x^{2}=V_{n}+V_{n-1}$ for some positive integer $x$. Firstly, let $k \equiv$ $1,5,7(\bmod 8)$ and $n>2$. We divide the reminder of the proof into four cases.

Case 1: Assume that $n=4 q-1$ with $q>0$. Then $n=2 \cdot 2^{r} a-1$ with $a$ odd and $r \geq 1$. By (2.4) and (2.3), it follows that

$$
x^{2}=V_{n}+V_{n-1} \equiv-\left(V_{-1}+V_{-2}\right) \equiv-\left(k^{2}+k-2\right)\left(\bmod V_{2^{r}}\right)
$$

and

$$
x^{2}=V_{n}+V_{n-1} \equiv V_{-1}+V_{-2} \equiv k^{2}+k-2 \equiv-2\left(\bmod U_{2}\right) .
$$

The above congruences give $\left(\frac{-\left(k^{2}+k-2\right)}{V_{2} r}\right)=1$ and $\left(\frac{-2}{k}\right)=1$. Since $\left(\frac{-2}{k}\right)=1$, we obtain $k \equiv 1,3(\bmod 8)$ by $(1.5)$. Since $k \equiv 1,5,7(\bmod 8)$, it follows that $k \equiv$ $1(\bmod 8)$. By $(2.6),(2.5)$, and $(2.7)$, we get

$$
1=\left(\frac{-\left(k^{2}+k-2\right)}{V_{2^{r}}}\right)=\left(\frac{-1}{V_{2^{r}}}\right)\left(\frac{k+2}{V_{2^{r}}}\right)\left(\frac{k-1}{V_{2^{r}}}\right)=(-1) \cdot 1 \cdot 1=-1,
$$

a contradiction.

Case 2: Assume that $n=4 q$ with $q \geq 1$. Then $n=2 \cdot 2^{r} a$ with $a$ odd and $r \geq 1$. By (2.4), we get

$$
x^{2}=V_{n}+V_{n-1} \equiv-\left(V_{0}+V_{-1}\right) \equiv-(k+2)\left(\bmod V_{2^{r}}\right) .
$$

This shows that $\left(\frac{-(k+2)}{V_{2} r}\right)=1$. Then by $(2.5)$, it follows that $\left(\frac{-(k+2)}{V_{2^{r}}}\right)$ $=\left(\frac{-1}{V_{2^{r}}}\right)\left(\frac{k+2}{V_{2^{r}}}\right)=(-1)\left(\frac{k+2}{V_{2^{r}}}\right)=1$, i.e., $\left(\frac{k+2}{V_{2^{r}}}\right)=-1$. Moreover, since

$$
x^{2}=V_{4 q}+V_{4 q-1} \equiv V_{0}+V_{-1} \equiv k+2 \equiv 2\left(\bmod U_{2}\right),
$$

we obtain $\left(\frac{2}{U_{2}}\right)=\left(\frac{2}{k}\right)=1$. By (1.4), we get $k \equiv 1,7(\bmod 8)$. Then by (2.7), it follows that $\left(\frac{k+2}{V_{2^{r}}}\right)=1$, which contradicts the fact that $\left(\frac{k+2}{V_{2^{r}}}\right)=-1$.

Case 3: Assume that $n=4 q+1$ with $q>0$. Then $n=2 \cdot 2^{r} a+1$ with $a$ odd and $r \geq 1$. By (2.4) and (2.3), it follows that

$$
x^{2}=V_{n}+V_{n-1} \equiv-\left(V_{1}+V_{0}\right) \equiv-(k+2)\left(\bmod V_{2^{r}}\right)
$$

and

$$
x^{2}=V_{4 q+1}+V_{4 q} \equiv V_{1}+V_{0} \equiv k+2 \equiv 2\left(\bmod U_{2}\right) .
$$

Both the congruences above give $\left(\frac{-(k+2)}{V_{2} r}\right)=1$ and $\left(\frac{2}{U_{2}}\right)=\left(\frac{2}{k}\right)=1$. Then by (1.4) and (2.7), we have a contradiction.

Case 4: Assume that $n=4 q+2$ for some $q>0$. Then $n=2 \cdot 2^{r} a+2$ with $a$ odd and $r \geq 1$. By (2.4) and (2.3), it follows that

$$
x^{2}=V_{n}+V_{n-1} \equiv-\left(V_{2}+V_{1}\right) \equiv-\left(k^{2}+k-2\right)\left(\bmod V_{2^{r}}\right)
$$

and

$$
x^{2}=V_{n}+V_{n-1} \equiv V_{2}+V_{1} \equiv-2\left(\bmod U_{2}\right) .
$$


These show that $\left(\frac{-\left(k^{2}+k-2\right)}{V_{2} r}\right)=1$ and $\left(\frac{-2}{k}\right)=1$. Since $\left(\frac{-2}{k}\right)=1$, we obtain $k \equiv$ $1,3(\bmod 8)$ by $(1.5)$. It follows that $k \equiv 1(\bmod 8)$ since $k \equiv 1,5,7(\bmod 8)$. By $(2.6)$, (2.5), and (2.7), we get

$$
1=\left(\frac{-\left(k^{2}+k-2\right)}{V_{2^{r}}}\right)=\left(\frac{-1}{V_{2^{r}}}\right)\left(\frac{k+2}{V_{2^{r}}}\right)\left(\frac{k-1}{V_{2^{r}}}\right)=(-1) \cdot 1 \cdot 1=-1,
$$

a contradiction. Thus we get $n \leq 2$. If $n=2$, then we obtain $x^{2}=V_{2}+V_{1}=$ $k^{2}+k-2$. The case that $k=2$ is impossible since $k \geq 3$. Thus we get $n=0$ or 1 .

Now, assume that $k \equiv 3(\bmod 8)$. Let $n=6 q+r$ with $0 \leq r \leq 5$. Then we get

$$
V_{n}=V_{6 q+r} \equiv V_{r}\left(\bmod U_{3}\right)
$$

by using (2.3). Since $8 \mid U_{3}$, it follows that $V_{n} \equiv V_{r}(\bmod 8)$ with $0 \leq r \leq 5$ and since $k \equiv 3(\bmod 8)$, it can be easily seen that $V_{n} \equiv V_{0}, V_{1}, V_{2}, V_{3}, V_{4}, V_{5} \equiv 2,3,7(\bmod 8)$. Then we obtain $x^{2}=V_{n}+V_{n-1} \equiv 5,2,1(\bmod 8)$. Since $x^{2} \equiv 0,1,4$ $(\bmod 8)$, we get $r=3$ or 4 .

Assume that $r=3$. Then $n=6 q+3$ with $q \geq 0$. Let $q$ be odd. Then by (2.4), it follows that

$$
x^{2}=V_{n}+V_{n-1}=V_{6 q+3}+V_{6 q+2} \equiv-\left(V_{3}+V_{2}\right) \equiv-V_{2} \equiv-\left(k^{2}-2\right)\left(\bmod V_{3}\right) \text {. }
$$

Since $V_{3}=k\left(k^{2}-3\right)$, we obtain

$$
x^{2} \equiv-\left(k^{2}-2\right) \equiv-k^{2}+2 \equiv-1\left(\bmod k^{2}-3\right) .
$$

This is impossible since $\frac{k^{2}-3}{2} \equiv-1(\bmod 4)$.

Let $q$ be even and $q>0$. Then $n=6 q+3=2 \cdot 2^{r} a+3$ with $a$ odd and $r \geq 1$. By (2.4), it follows that

$$
\begin{aligned}
x^{2}=V_{n}+V_{n-1}=V_{2 \cdot 2^{r} a+3} & +V_{2 \cdot 2^{r} a+2} \\
& \equiv-\left(V_{3}+V_{2}\right) \equiv-(k+2)\left(k^{2}-k-1\right)\left(\bmod V_{2^{r}}\right) .
\end{aligned}
$$

This shows that $\left(\frac{-(k+2)\left(k^{2}-k-1\right)}{V_{2} r}\right)=1$. However, this is impossible since

$$
\left(\frac{-(k+2)\left(k^{2}-k-1\right)}{V_{2^{r}}}\right)=\left(\frac{-1}{V_{2^{r}}}\right)\left(\frac{k+2}{V_{2^{r}}}\right)\left(\frac{k^{2}-k-1}{V_{2^{r}}}\right)=(-1)(-1)(-1)=-1
$$

by (2.5), (2.7), and Lemma 2. If $q=0$, then we get $x^{2}=V_{3}+V_{2}=k^{3}+k^{2}-3 k-$ 2. But the integer points on $Y^{2}=X^{3}+X^{2}-3 X-2$ are easily determined by using MAGMA [1] to be $(X, Y)=(-2,0),(-1, \mp 1),(3, \mp 5),(2, \mp 2),(43, \mp 285)$. Then it follows that $k=3$ or 43 .

Now, assume that $r=4$. Then $n=6 q+4$ with $q \geq 0$. Thus we can write $n=$ $6(q+1)-2=6 t-2$ and therefore,

$$
V_{n}+V_{n-1}=V_{6 t-2}+V_{6 t-3} \equiv-\left(V_{3}+V_{2}\right) \equiv-V_{2} \equiv-\left(k^{2}-2\right)\left(\bmod V_{3}\right) .
$$


Since $V_{3}=k\left(k^{2}-3\right)$, we obtain

$$
x^{2} \equiv-\left(k^{2}-2\right) \equiv-k^{2}+2 \equiv-1\left(\bmod k^{2}-3\right) .
$$

This is impossible since $\frac{k^{2}-3}{2} \equiv-1(\bmod 4)$.

Theorem 12. Let $k \equiv 3,5,7(\bmod 8)$. If the equation $x^{2}=V_{n}-V_{n-1}$ has a solution, then $n=1$ or 2 . Moreover, if $k \equiv 1(\bmod 8)$, then the equation $x^{2}=V_{n}-V_{n-1}$ has no solutions.

Proof. Let $k \equiv 3,5,7(\bmod 8)$. Assume that $x^{2}=V_{n}-V_{n-1}$ for some positive integer $x$. Let $n>2$. We divide the reminder of the proof into four cases.

Case 1: Assume that $n=4 q-1$ with $q>0$. Then $n=2 \cdot 2^{r} a-1$ with $a$ odd and $r \geq 1$. By (2.4) and (2.3), it follows that

$$
x^{2}=V_{4 q-1}-V_{4 q-2} \equiv-\left(V_{-1}-V_{-2}\right) \equiv k^{2}-k-2\left(\bmod V_{2^{r}}\right)
$$

and

$$
x^{2}=V_{4 q-1}-V_{4 q-2} \equiv V_{-1}-V_{-2} \equiv 2\left(\bmod U_{2}\right) .
$$

These show that $\left(\frac{k^{2}-k-2}{V_{2} r}\right)=1$ and $\left(\frac{2}{k}\right)=1$. Since $\left(\frac{2}{k}\right)=1$, it follows that $k \equiv$ $1,7(\bmod 8)$ by $(1.4)$. Since $k \equiv 3,5,7(\bmod 8)$, we get $k \equiv 7(\bmod 8)$. Then by using (1.4) and (2.8), we obtain

$$
1=\left(\frac{k^{2}-k-2}{V_{2^{r}}}\right)=\left(\frac{k-2}{V_{2^{r}}}\right)\left(\frac{k+1}{V_{2^{r}}}\right)=(-1) \cdot 1=-1,
$$

a contradiction.

Case 2: Assume that $n=4 q$ with $q \geq 1$. Then $n=2 \cdot 2^{r} a$ with $a$ odd and $r \geq 1$. By (2.4), we get

$$
x^{2}=V_{4 q}-V_{4 q-1} \equiv-\left(V_{0}-V_{-1}\right) \equiv k-2\left(\bmod V_{2^{r}}\right) .
$$

This shows that $\left(\frac{k-2}{V_{2} r}\right)=1$. On the other hand, by using (2.3), we obtain

$$
x^{2}=V_{4 q}-V_{4 q-1} \equiv V_{0}-V_{-1} \equiv-k+2 \equiv 2\left(\bmod U_{2}\right),
$$

which implies that $\left(\frac{2}{k}\right)=1$. Then it follows that $k \equiv 1,7(\bmod 8)$ by $(1.4)$. Since $k \equiv 3,5,7(\bmod 8)$, we have $k \equiv 7(\bmod 8)$. Then by $(2.8)$, we get $\left(\frac{k-2}{V_{2} r}\right)=-1$, which contradicts the fact that $\left(\frac{k-2}{V_{2} r}\right)=1$.

Case 3: Assume that $n=4 q+1$ with $q>0$. Then $n=2 \cdot 2^{r} a+1$ with $a$ odd and $r \geq 1$. By (2.4) and (2.3), it follows that

$$
x^{2}=V_{4 q+1}-V_{4 q} \equiv-\left(V_{1}-V_{0}\right) \equiv-(k-2)\left(\bmod V_{2^{r}}\right)
$$

and

$$
x^{2}=V_{4 q+1}-V_{4 q} \equiv V_{1}-V_{0} \equiv-2\left(\bmod U_{2}\right) .
$$


These show that $\left(\frac{-(k-2)}{V_{2} r}\right)=1$ and $\left(\frac{-2}{k}\right)=1$. Since $\left(\frac{-2}{k}\right)=1$, it follows that $k \equiv$ $1,3(\bmod 8)$ by $(1.5)$. Since $k \equiv 3,5,7(\bmod 8)$, we get $k \equiv 3(\bmod 8)$. Then by $(2.5)$ and (2.8), we obtain

$$
\left(\frac{-(k-2)}{V_{2^{r}}}\right)=\left(\frac{-1}{V_{2^{r}}}\right)\left(\frac{k-2}{V_{2^{r}}}\right)=-1,
$$

which contradicts the fact that $\left(\frac{-(k-2)}{V_{2} r}\right)=1$.

Case 4: Assume that $n=4 q+2$ with $q>0$. Then $n=2 \cdot 2^{r} a+2$ with $a$ odd and $r \geq 1$. By (2.4) and (2.3), it follows that

$$
x^{2}=V_{4 q+2}-V_{4 q+1} \equiv-\left(V_{2}-V_{1}\right) \equiv-\left(k^{2}-k-2\right)\left(\bmod V_{2^{r}}\right)
$$

and

$$
x^{2}=V_{4 q+2}-V_{4 q+1} \equiv V_{2}-V_{1} \equiv-2\left(\bmod U_{2}\right) .
$$

The above congruences give $\left(\frac{-\left(k^{2}-k-2\right)}{V_{2} r}\right)=1$ and $\left(\frac{-2}{k}\right)=1$. Since $\left(\frac{-2}{k}\right)=1$, it follows that $k \equiv 1,3(\bmod 8)$ by $(1.5)$. Since $k \equiv 3,5,7(\bmod 8)$, we get $k \equiv 3(\bmod 8)$. Then by using (1.4), (2.5), and (2.8), we obtain $1=\left(\frac{-\left(k^{2}-k-2\right)}{V_{2} r}\right)=$ $\left(\frac{-1}{V_{2^{r}}}\right)\left(\frac{k-2}{V_{2^{r}}}\right)\left(\frac{k+1}{V_{2^{r}}}\right)=-1$, a contradiction. Therefore, $n \leq 2$. If $n=0$, then we have $x^{2}=V_{0}-V_{-1}=2-k$, which is impossible. So, we get $n=1$ or 2 .

Now, assume that $k \equiv 1(\bmod 8)$. Let $n=6 q+r$ with $0 \leq r \leq 5$. Then by using (2.3), we get

$$
V_{n}=V_{6 q+r} \equiv V_{r}\left(\bmod U_{3}\right) .
$$

Since $8 \mid U_{3}$, it follows that $V_{n} \equiv V_{r}(\bmod 8)$ with $0 \leq r \leq 5$. Moreover, since $k \equiv$ $1(\bmod 8)$, it can be easily seen that $V_{n} \equiv V_{0}, V_{1}, V_{2}, V_{3}, V_{4}, V_{5} \equiv 2,1,7,6(\bmod 8)$. Then we get $x^{2}=V_{n}-V_{n-1} \equiv 1,6,7(\bmod 8)$. It can be seen that $r=0$ or 4 .

Assume that $r=0$. Then $n=6 q$ with $q \geq 0$. Let $q$ be odd. Then it follows that

$$
x^{2}=V_{n}-V_{n-1}=V_{6 q}-V_{6 q-1} \equiv-\left(V_{0}-V_{-1}\right) \equiv k-2\left(\bmod V_{3}\right)
$$

by (2.4). Since $V_{3}=k\left(k^{2}-3\right)$, we obtain

$$
x^{2} \equiv k-2\left(\bmod k^{2}-3\right)
$$

which implies that $x^{2} \equiv k-2\left(\bmod \frac{k^{2}-3}{2}\right)$. Therefore, $\left(\frac{k-2}{\left(k^{2}-3\right) / 2}\right)=1$. Since $k \equiv$ $1(\bmod 8)$, we get $\frac{k^{2}-3}{2} \equiv 3(\bmod 4)$. These show that

$$
\begin{aligned}
1 & =\left(\frac{k-2}{\left(k^{2}-3\right) / 2}\right)=(-1)^{\left(\frac{k-3}{2}\right)\left(\frac{k^{2}-5}{4}\right)}\left(\frac{\left(k^{2}-3\right) / 2}{k-2}\right) \\
& =(-1)\left(\frac{2}{k-2}\right)\left(\frac{k^{2}-3}{k-2}\right)
\end{aligned}
$$




$$
=(-1) \cdot 1 \cdot\left(\frac{1}{k-2}\right)=-1,
$$

a contradiction. Let $q$ be even. Then $n=6 q=2 \cdot 2^{r} a$ with $a$ odd and $r \geq 1$. By (2.4), it follows that

$$
x^{2}=V_{n}-V_{n-1}=V_{2 \cdot 2^{r} a}-V_{2 \cdot 2^{r} a-1} \equiv-\left(V_{0}-V_{-1}\right) \equiv k-2\left(\bmod V_{2^{r}}\right) .
$$

This shows that $\left(\frac{k-2}{V_{2^{r}}}\right)=1$, which is impossible by (2.8).

Now, assume that $r=4$. Then $n=6 q+4$ with $q \geq 0$. We can write $n=6(q+$ 1) $-2=6 t-2$. Let $t$ be odd. Then it follows that

$$
x^{2}=V_{n}-V_{n-1}=V_{6 t-2}-V_{6 t-3} \equiv-\left(V_{-2}-V_{-3}\right) \equiv-V_{2} \equiv-\left(k^{2}-2\right)\left(\bmod V_{3}\right)
$$

by (2.4). Since $V_{3}=k\left(k^{2}-3\right)$, we obtain

$$
x^{2} \equiv-\left(k^{2}-2\right) \equiv-1\left(\bmod k^{2}-3\right),
$$

which shows that $x^{2} \equiv-1\left(\bmod \frac{k^{2}-3}{2}\right)$. But this is impossible since $\frac{k^{2}-3}{2} \equiv 3(\bmod 4)$. Let $t$ be even. Then $n=6 t-2=2 \cdot 2^{r} a-2$ with $a$ odd and $r \geq 1$. It follows that

$$
\begin{aligned}
x^{2} & =V_{n}-V_{n-1}=V_{2 \cdot 2^{r} a-2}-V_{2 \cdot 2^{r} a-3} \\
& \equiv-\left(V_{-2}-V_{-3}\right) \equiv(k-2)\left(k^{2}+k-1\right)\left(\bmod V_{2^{r}}\right)
\end{aligned}
$$

by (2.4). This shows that $\left(\frac{(k-2)\left(k^{2}+k-1\right)}{V_{2} r}\right)=1$. Then by (2.8) and Lemma 4, we obtain

$$
\left(\frac{k-2}{V_{2^{r}}}\right)\left(\frac{k^{2}+k-1}{V_{2^{r}}}\right)=-1,
$$

which contradicts the fact that $\left(\frac{(k-2)\left(k^{2}+k-1\right)}{V_{2} r}\right)=1$. This completes the proof.

Theorem 13. If the equation $x^{2}=U_{n+1}+U_{n}$ has a solution, then $n=0$ or 1 .

Proof. Assume that $x^{2}=U_{n+1}+U_{n}$ for some positive integer $x$. Now, assume that $n>2$. We distinquish four cases.

Case 1: Assume that $n=4 q-1$ with $q>0$. Then by (2.1), we get

$$
x^{2}=U_{4 q}+U_{4 q-1} \equiv U_{0}+U_{-1} \equiv-1\left(\bmod U_{2}\right) .
$$

This shows that $\left(\frac{-1}{k}\right)=1$. Then it follows that $k \equiv 1,5(\bmod 8)$ by $(1.3)$.

Let $q=3 u-1$ with $u>0$. Then $n=12 u-5$. By using (2.1), we obtain

$$
x^{2}=U_{12 u-4}+U_{12 u-5} \equiv U_{-4}+U_{-5} \equiv k-3\left(\bmod U_{3}\right) .
$$

Since $8 \mid U_{3}$, we get $x^{2} \equiv k-3(\bmod 8)$. Moreover, since $k \equiv 1,5(\bmod 8)$, it follows that $x^{2} \equiv-2,2(\bmod 8)$, which is impossible.

Let $q=3 u$ with $u>0$. Then $n=12 u-1$. Here, we get

$$
x^{2}=U_{12 u-1}+U_{12 u} \equiv U_{-1}+U_{0} \equiv-1\left(\bmod U_{3}\right)
$$


by (2.1). Since $8 \mid U_{3}$, it follows that $x^{2} \equiv-1(\bmod 8)$, which is impossible.

Let $q=3 u+1$ with $u \geq 0$. Then $n=12 u+3$. Here, we obtain

$$
x^{2}=U_{12 u+4}+U_{12 u+3} \equiv U_{4}+U_{3} \equiv U_{4} \equiv-k\left(\bmod U_{3}\right)
$$

by (2.1). Since $8 \mid U_{3}$, it follows that $x^{2} \equiv-k(\bmod 8)$. Moreover, since $k \equiv$ $1,5(\bmod 8)$, we obtain $x^{2} \equiv-1,-5(\bmod 8)$. However, this is impossible.

Case 2: Assume that $n=4 q$ with $q \geq 1$. Then $n=2 \cdot 2^{r} a$ with $a$ odd and $r \geq 1$. By (2.2), we get

$$
x^{2}=U_{4 q+1}+U_{4 q} \equiv-\left(U_{1}+U_{0}\right) \equiv-1\left(\bmod V_{2^{r}}\right),
$$

which is impossible by (2.5).

Case 3: Assume that $n=4 q+1$ with $q>0$. Then $n=2 \cdot 2^{r} a+1$ with $a$ odd and $r \geq 1$. By (2.2), we get

$$
x^{2}=U_{4 q+2}+U_{4 q+1} \equiv-\left(U_{2}+U_{1}\right) \equiv-(k+1)\left(\bmod V_{2} r\right) .
$$

This shows that $\left(\frac{-(k+1)}{V_{2^{r}}}\right)=1$. Since $\left(\frac{-1}{V_{2^{r}}}\right)=-1$ and $\left(\frac{-(k+1)}{V_{2^{r}}}\right)=\left(\frac{-1}{V_{2^{r}}}\right)\left(\frac{k+1}{V_{2^{r}}}\right)$, it follows that $\left(\frac{k+1}{V_{2} r}\right)=-1$. This is impossible by (1.5).

Case 4: Assume that $n=4 q+2$. Then $n=2 \cdot 2^{r} a+2$ with $a$ odd and $r \geq 1$. By (2.1), we obtain

$$
x^{2}=U_{4 q+3}+U_{4 q+2} \equiv U_{3}+U_{2} \equiv-1\left(\bmod U_{2}\right),
$$

i.e., $\left(\frac{-1}{k}\right)=1$. It follows that $k \equiv 1,5(\bmod 8)$ by (1.3). On the other hand, by (2.2), we get

$$
x^{2}=U_{4 q+3}+U_{4 q+2} \equiv-\left(U_{3}+U_{2}\right) \equiv-\left(k^{2}+k-1\right)\left(\bmod V_{2^{r}}\right) .
$$

This shows that $\left(\frac{-\left(k^{2}+k-1\right)}{V_{2} r}\right)=1$. Since $\left(\frac{-\left(k^{2}+k-1\right)}{V_{2} r}\right)=\left(\frac{-1}{V_{2} r}\right)\left(\frac{k^{2}+k-1}{V_{2} r}\right)=1$ and $\left(\frac{-1}{V_{2^{r}}}\right)=-1$, we have $\left(\frac{k^{2}+k-1}{V_{2^{r}}}\right)=-1$. This is impossible by Lemma 4 . Therefore, $n \leq 2$. If $n=2$, then $x^{2}=U_{3}+U_{2}=k^{2}+k-1$, which shows that $k=1$. This is impossible since $k \geq 3$. Thus we get $n=0$ or 1 .

Since the proof of the following theorem is similar to that of above theorem, we omit its proof.

Theorem 14. If the equation $x^{2}=U_{n+1}-U_{n}$ has a solution, then $n=0$ or 1 .

\section{MAIN THeOREMS}

Now, we consider positive integer solutions of some fourth-order Diophantine equations. 
Theorem 15. Let $k+2$ be not a perfect square. If $k+1$ is a perfect square, then all positive integer solutions of the equation $x^{4}-k x^{2} y+y^{2}=k+2$ are given by $(x, y)=(\sqrt{k+1}, 1),\left(\sqrt{k+1}, k^{2}+k-1\right),(1, k+1)$. If $k+1$ is not a perfect square, then the only positive integer solution of this equation is $(x, y)=(1, k+1)$.

Proof. Assume that $x^{4}-k x^{2} y+y^{2}=k+2$ for some positive integers $x$ and $y$. Then by Theorem 4 , we obtain $x^{2}=U_{n+1}+U_{n}, y=U_{n}+U_{n-1}$ or $x^{2}=U_{n}+$ $U_{n-1}, y=U_{n+1}+U_{n}$ with $n>0$. Let $x^{2}=U_{n+1}+U_{n}$. Then it follows that $n=0$ or 1 by Theorem 13. Since $n>0$, we have $n=1$. Then we have $x^{2}=U_{n+1}+$ $U_{n}=U_{2}+U_{1}=k+1$ and $y=U_{1}+U_{0}=1$. If $k+1$ is a perfect square, then it follows that $x=\sqrt{k+1}$ and $y=1$. Now, let $x^{2}=U_{n}+U_{n-1}$. Then we obtain $n-1=0$ or 1 by Theorem 13. Assume that $n=1$. Then $x^{2}=U_{1}+U_{0}=1$ and $y=U_{2}+U_{1}=k+1$. Thus we have $x=1$ and $y=k+1$. Now, assume that $n=2$. Then we get $x^{2}=U_{2}+U_{1}=k+1$ and $y=U_{3}+U_{2}=k^{2}+k-1$. If $k+1$ is a perfect square, then it follows that $x=\sqrt{k+1}$ and $y=k^{2}+k-1$. Thus if $k+1$ is not a perfect square, then this equation has no positive integer solutions. Then the proof follows.

Theorem 16. If $k-1$ is a perfect square, then all positive integer solutions of the equation $x^{4}-k x^{2} y+y^{2}=-(k-2)$ are given by $(x, y)=(1,1),(\sqrt{k-1}, 1)$, $\left(\sqrt{k-1}, k^{2}-k-1\right),(1, k-1)$. Moreover, if $k-1$ is not a perfect square, then all positive integer solutions of this equation are given by $(x, y)=(1,1)$ or $(1, k-1)$.

Proof. Assume that $x^{4}-k x^{2} y+y^{2}=-(k-2)$ for some positive integers $x$ and $y$. Then by Theorem 5 , we obtain $x^{2}=U_{n+1}-U_{n}, y=U_{n}-U_{n-1}$ or $x^{2}=U_{n}-$ $U_{n-1}, y=U_{n+1}-U_{n}$ with $n \geq 0$. Let $x^{2}=U_{n+1}-U_{n}$. Then it follows that $n=0$ or 1 by Theorem 14 . Assume that $n=0$. Then we have $x^{2}=U_{n+1}-U_{n}=U_{1}-U_{0}=1$ and $y=U_{0}-U_{-1}=1$. Thus we have $x=1$ and $y=1$. Now, assume that $n=1$. Then we get $x^{2}=U_{2}-U_{1}=k-1$ and $y=U_{1}-U_{0}=1$. If $k-1$ is a perfect square, then it follows that $x=\sqrt{k-1}$ and $y=1$. Now, let $x^{2}=U_{n}-U_{n-1}$. Then we obtain $n=1$ or 2 by Theorem 14 . Assume that $n=1$. Then $x^{2}=U_{1}-U_{0}=1$ and $y=U_{2}-U_{1}=k-1$. Thus we have $x=1$ and $y=k-1$. Now, assume that $n=2$. Then we get $x^{2}=U_{2}-U_{1}=k-1$ and $y=U_{3}-U_{2}=k^{2}-k-1$. If $k-1$ is a perfect square, then it follows that $x=\sqrt{k-1}$ and $y=k^{2}-k-1$. Thus if $k-1$ is not a perfect square, then this equation has no positive integer solutions other than $(1,1)$ and $(1, k-1)$.

Theorem 17. If $k \equiv 1,5,7(\bmod 8)$, then the equation $x^{4}-\left(k^{2}-4\right) y^{2}=4(k+2)$ has a solution if and only if $k+2$ is a perfect square. Moreover, if $k \equiv 1,5,7(\bmod 8)$ and $k+2$ is a perfect square, then the only positive integer solution of this equation is $(x, y)=(\sqrt{k+2}, 1)$. If $k \equiv 3(\bmod 8)$, then the equation $x^{4}-\left(k^{2}-4\right) y^{2}=4(k+2)$ has positive integer solutions only when $k=3$ or 43 . 
Proof. Assume that $x^{4}-\left(k^{2}-4\right) y^{2}=4(k+2)$ for some positive integers $x$ and $y$. Firstly, let $k \equiv 1,5,7(\bmod 8)$ and $k+2$ be not a perfect square. Then by Theorem 6 , it follows that $x^{2}=V_{n}+V_{n-1}$ with $n \geq 1$. Therefore, by Theorem 11, it is seen that $n=0$ or 1 . Since $n \geq 1$, we get $n=1$. Then it follows that $x^{2}=k+2$. But this is impossible since $k+2$ is not a perfect square. Let $k \equiv 1,5,7(\bmod 8)$ and $k+2$ be a perfect square. Then by Theorem 7, it follows that $x^{2}=\sqrt{k+2} V_{n-2}$. Therefore, we obtain $V_{n-2}(\sqrt{k+2},-1)=\square, \sqrt{k+2} \square$ or $a \square$ with $a \mid \sqrt{k+2}$. If $V_{n-2}(\sqrt{k+2},-1)=\sqrt{k+2} \square$ or $a \square$ with $a \mid \sqrt{k+2}$, then it follows that $n=3$ by Theorem 2 and Theorem 1 . Then it can be seen that $x=\sqrt{k+2}$ and $y=1$. If $V_{n-2}(\sqrt{k+2},-1)=\square$, we have $n=3$ and $k+2$ is a perfect square by Lemma 1 . Thus if $k+2$ is a perfect square, then we obtain $x=\sqrt{k+2}$ and $y=1$. Now, let $k \equiv 3(\bmod 8)$. Then $k+2$ is not a perfect square and it follows that $k=3$ or 43 by Theorem 6 and Theorem 11. When $k=3$, we get the solution $(x, y)=(5,11)$. When $k=43$, we get the solution $(x, y)=(285,1891)$.

Theorem 18. If $k+1$ is not a perfect square, then the only positive integer solution of the equation $x^{2}-\left(k^{2}-4\right) y^{4}=4(k+2)$ is $(x, y)=(k+2,1)$. Moreover, if $k+1$ is a perfect square, then all positive integer solutions of this equation are given by $(x, y)=\left(k^{2}+k-2, \sqrt{k+1}\right)$ or $(k+2,1)$.

Proof. Assume that $x^{2}-\left(k^{2}-4\right) y^{4}=4(k+2)$ for some positive integers $x$ and $y$. Then by Theorem 6 , it follows that $x=V_{n}+V_{n-1}, y^{2}=U_{n}+U_{n-1}$ with $n>0$. Therefore, by Theorem 13, we have $n=1$ or 2 . Let $n=1$. Then it follows that $x=V_{1}+V_{0}=k+2$ and $y^{2}=U_{1}+U_{0}=1$. Therefore, we obtain $(x, y)=(k+2,1)$. Let $n=2$. Then it is seen that $x=V_{2}+V_{1}=k^{2}-2+k$ and $y^{2}=U_{2}+U_{1}=k+1$. If $k+1$ is a perfect square, then we obtain $x=k^{2}+k-2$ and $y=\sqrt{k+1}$.

Theorem 19. Let $k \equiv 3,5,7(\bmod 8)$. If $k=3$, then all positive integer solutions of the equation $x^{4}-\left(k^{2}-4\right) y^{2}=-4(k-2)$ are given by $(x, y)=(2,2),(1,1)$. If $k \neq 3$ and $k-2$ is a perfect square, then the only positive integer solution of the equation $x^{4}-\left(k^{2}-4\right) y^{2}=-4(k-2)$ is $(x, y)=(\sqrt{k-2}, 1)$. If $k \neq 3$ and $k-2$ is not a perfect square, then this equation has no positive integer solutions.

Proof. Assume that $x^{4}-\left(k^{2}-4\right) y^{2}=-4(k-2)$ for some positive integers $x$ and $y$. Then by Theorem 8 , we obtain $x^{2}=V_{n}-V_{n-1}$ and $y=U_{n}-U_{n-1}$ with $n>0$. Since $k \equiv 3,5,7(\bmod 8)$ and $x^{2}=V_{n}-V_{n-1}$, it follows that $n=1$ or 2 by Theorem 12. Let $n=1$. Then we have $x^{2}=V_{1}-V_{0}=k-2$ and $y=U_{1}-U_{0}=1$. If $k-2$ is a perfect square, then we obtain $(x, y)=(\sqrt{k-2}, 1)$. Otherwise this equation has no solutions. Let $n=2$. Then it follows that $x^{2}=V_{2}-V_{1}=k^{2}-2-k$ and $y=U_{2}-U_{1}=k-1$. Since $x^{2}=k^{2}-k-2$, we get $4 x^{2}=4 k^{2}-4 k-8$, i.e., $(2 k-1)^{2}-(2 x)^{2}=9$. This shows that $k=3$. Hence, we get $x=2$ and $y=2$.

Theorem 20. Let $k \equiv 1(\bmod 8)$. Then the equation $x^{4}-\left(k^{2}-4\right) y^{2}=-4(k-2)$ has no integer solutions. 
Proof. Assume that $x^{4}-\left(k^{2}-4\right) y^{2}=-4(k-2)$ for some positive integers $x$ and $y$. Then by Theorem 8 , we obtain $x^{2}=V_{n}-V_{n-1}$ and $y=U_{n}-U_{n-1}$. This is impossible by Theorem 12 .

Theorem 21. If $k-1$ is not a perfect square, then the only positive integer solution of the equation $x^{2}-\left(k^{2}-4\right) y^{4}=-4(k-2)$ is $(x, y)=(k-2,1)$. Moreover, if $k-1$ is a perfect square, then all positive integer solutions of the equation $x^{2}-\left(k^{2}-\right.$ 4) $y^{4}=-4(k-2)$ are given by $(x, y)=\left(k^{2}-2-k, \sqrt{k-1}\right),(k-2,1)$.

Proof. Assume that $x^{2}-\left(k^{2}-4\right) y^{4}=-4(k-2)$ for some positive integers $x$ and $y$. Then by Theorem 8, we obtain $x=V_{n}-V_{n-1}$ and $y^{2}=U_{n}-U_{n-1}$ with $n>0$. Therefore, by Theorem 14, it follows that $n=1$ or 2 . Let $n=1$. Then we have $x=$ $V_{1}-V_{0}=k-2$ and $y^{2}=U_{1}-U_{0}=1$. Therefore, $(x, y)=(k-2,1)$. Now, let $n=2$. Then it follows that $x=V_{2}-V_{1}=k^{2}-2-k$ and $y^{2}=U_{2}-U_{1}=k-1$. Therefore, if $k-1$ is a perfect square, then it follows that $(x, y)=\left(k^{2}-k-2, \sqrt{k-1}\right)$ is a solution.

Theorem 22. Let $k \equiv 1,5,7(\bmod 8)$ and $k^{2}-4$ be square-free. Then the equation $x^{4}-k x^{2} y+y^{2}=-(k+2)\left(k^{2}-4\right)$ has no positive integer solutions.

Proof. Assume that $x^{4}-k x^{2} y+y^{2}=-(k+2)\left(k^{2}-4\right)$ for some positive integers $x$ and $y$. Then by Theorem 9, we obtain $x^{2}=V_{n+1}+V_{n}, y=V_{n}+V_{n-1}$ or $x^{2}=V_{n}+V_{n-1}, y=V_{n+1}+V_{n}$ with $n \geq 0$. Let $x^{2}=V_{n+1}+V_{n}$. Since $k \equiv$ $1,5,7(\bmod 8)$, we obtain $n+1=0$ or 1 by Theorem 11 . Since $n \geq 0$, we get $n=0$. Then we have $x^{2}=V_{1}+V_{0}=k+2$ and $y=V_{0}+V_{-1}=k+2$. This is impossible since $k^{2}-4$ is square-free. Let $x^{2}=V_{n}+V_{n-1}$. Then by Theorem 11 , we obtain $n=0$ or 1 . Thus it follows that $x^{2}=V_{0}+V_{-1}=V_{1}+V_{0}=k+2$. This is impossible since $k^{2}-4$ is square-free.

From Theorem 9 and Theorem 11, we can give the following theorem.

Theorem 23. Let $k \equiv 3(\bmod 8)$ and $k^{2}-4$ be square-free. Then the equation $x^{4}-k x^{2} y+y^{2}=-(k+2)\left(k^{2}-4\right)$ has no positive integer solutions.

Theorem 24. Let $k \equiv 3,5,7(\bmod 8)$ and $k^{2}-4$ be square-free. Then the equation $x^{4}-k x^{2} y+y^{2}=(k-2)\left(k^{2}-4\right)$ has positive integer solutions only when $k=3$ and in which case all positive integer solutions are given by $(x, y)=(2,1),(1,4)$ or $(2,11)$.

Proof. Assume that $x^{4}-k x^{2} y+y^{2}=(k-2)\left(k^{2}-4\right)$ for some positive integers $x$ and $y$. Then by Theorem 10, we obtain $x^{2}=V_{n+1}-V_{n}, y=V_{n}-V_{n-1}$ or $x^{2}=V_{n}-$ $V_{n-1}, y=V_{n+1}-V_{n}$ with $n \geq 1$. Assume that $x^{2}=V_{n+1}-V_{n}$. Then by Theorem 12 , we obtain $n+1=1$ or 2 . Since $n \geq 1$, we get $n=1$. Then it follows that $x^{2}=$ $V_{2}-V_{1}=k^{2}-k-2$ and $y=V_{1}-\bar{V}_{0}=k-2$. Since $x^{2}=k^{2}-k-2$, we get $4 x^{2}=4 k^{2}-4 k-8$, i.e., $(2 k-1)^{2}-(2 x)^{2}=9$. Thus it follows that $k=3$. Then it can be seen that $(x, y)=(2,1)$. Now, assume that $x^{2}=V_{n}-V_{n-1}$. Then by Theorem 
12 , it follows that $n=1$ or 2 . Let $n=1$. Then we get $x^{2}=V_{1}-V_{0}=k-2$ and $y=V_{2}-V_{1}=k^{2}-2-k$. Thus $k-2$ must be a perfect square. But this is impossible in the case that $k>3$. Let $k=3$. Then we obtain $x=1$ and $y=4$. Let $n=2$. Then we obtain $x^{2}=V_{2}-V_{1}=k^{2}-k-2$. It can be shown that $k=3$. Then we have $(x, y)=(2,11)$. This completes the proof.

From Theorem 10 and Theorem 12, we can give the following theorem.

Theorem 25. Let $k \equiv 1(\bmod 8)$ and $k^{2}-4$ be square-free. Then the equation $x^{4}-k x^{2} y+y^{2}=(k-2)\left(k^{2}-4\right)$ has no positive integer solutions.

\section{ACKNOWLEDGEMENT}

Thanks to The Scientific and Technological Research Council of Turkey (TÜBİTAK) for support and assistance.

\section{REFERENCES}

[1] W. Bosma, J. Cannon, and C. Playoust, "The MAGMA algebra system I. The user language," J. Symbolic Comput., vol. 24, no. 3-4, pp. 235-265, 1997, doi: 10.1006/jsco.1996.0125.

[2] J. H. E. Cohn, “Twelve Diophantine Equations," Arch. Math., vol. 65, no. 2, pp. 130-133, 1995, doi: 10.1007/BF01270690.

[3] R. Keskin, "Generalized Fibonacci and Lucas Numbers of the form $w x^{2}$ and $w x^{2} \pm 1$, ," Bull. Korean Math. Soc., vol. 51, no. 4, pp. 1041-1054, 2014, doi: 10.4134/BKMS.2014.51.4.1041.

[4] R. Keskin and M. Güney Duman, "Positive integer solutions of some second-order Diophantine Equations," An. ŞtiinCuza Iaşi. Mat., (accepted), 2017.

[5] P. Ribenboim and W. L. McDaniel, "The square terms in Lucas sequences," J. Number Theory, vol. 58, no. 1, pp. 104-123, 1996, doi: 10.1006/jnth.1996.0068.

[6] Z. Şiar and R. Keskin, "Some new identities concerning generalized Fibonacci and Lucas numbers," Hacet. J. Math. Stat., vol. 42, no. 3, pp. 211-222, 2013, doi: 10.1142/S1793042115500517.

[7] G. Walsh, "A note on a theorem of Ljunggren and the Diophantine equations $x^{2}-k x y^{2}+y^{4}=$ 1,4," Arch. Math., vol. 73, no. 2, pp. 119-125, 1999, doi: 10.1007/s000130050376.

Authors' addresses

Refik Keskin

Sakarya University, Mathematics Department, TR 54187 Sakarya, Turkey

E-mail address: rkeskin@sakarya.edu.tr

Merve Güney Duman

Sakarya University, Mathematics Department, TR 54187 Sakarya, Turkey

E-mail address: merveguneyduman@gmail.com 